McGill-95/2

hep-ph/9503489

January 1995

\title{
Heavy-Quark Production by Polarized and Unpolarized Photons in Next-to-Leading Order
}

\author{
B. Kamal, ${ }^{a, 1}$ Z. Merebashvili ${ }^{a, 2, *}$ and A.P. Contogouris ${ }^{a, b, 3}$ \\ a. Department of Physics, McGill University, Montreal, Qc., H3A 2T8, Canada \\ b. Nuclear and Particle Physics, University of Athens, Athens 15771, Greece
}

\begin{abstract}
Complete analytical results for the production of heavy-quark pairs by polarized and unpolarized photons in next-to-leading order are presented. Two-, three- and two plus three-jet cross sections for total photon spin $J_{z}=0, \pm 2$ are presented for $b \bar{b}(g)$ production. The 2-jet cross sections are considered as a background to $\gamma \gamma \rightarrow H^{*} \rightarrow b \bar{b}$ (standard model). Top production, not too far above threshold, is also considered for $J_{z}=0, \pm 2$. For both $b$ and $t$-quark production, the higher order QCD corrections are found to be significant.
\end{abstract}

(Phys. Rev. D 51, 4808 (1995))

\footnotetext{
${ }^{1}$ e-mail: kamal@wind.phy.bnl.gov

${ }^{2}$ e-mail: zaza@hep.physics.mcgill.ca

${ }^{3}$ e-mail: apcont@hep.physics.mcgill.ca, acontog@atlas.uoa.ariadne-t.gr
} 


\section{INTRODUCTION}

Higher (next-to-leading) order corrections (HOC) for heavy-quark $(Q, \bar{Q})$ production in unpolarized particle collisions have been determined in detail. 1 , 3 For polarized particle collisions, however, analytical results were still absent. Even for the unpolarized case, only virtual + soft corrections have been presented analytically. 2 Apart from general reasons, well known from unpolarized reactions, knowledge of HOC for $Q, \bar{Q}$ production in polarized processes is important for several special reasons.

Beginning with polarized $\gamma \gamma$ collisions, which is the subject of the present work, one reason of special interest is the following. A $\gamma \gamma$ collider becomes particularly important for searches of the standard model Higgs boson when its mass is below the $W^{+} W^{-}$threshold. Then the predominant decay is $H \rightarrow b \bar{b}$ and the background comes from $\gamma \gamma \rightarrow b \bar{b}$ with direct or resolved photons. Leaving aside the latter, for the moment, use of polarized photons of equal helicity (when the angular momentum has $J_{z}=0$ ) suppresses this background by a factor $m_{b}^{2} / s$. 4 . This holds, however, only for the lowest order of $\alpha_{s}$. HOC necessarily involve the subprocess $\gamma \gamma \rightarrow b \bar{b} g$, and gluon emission permits the $b \bar{b}$ system to have $J \neq 0$ without suppression; this may result in a sizable background. Of course, another reason the $J_{z}=0$ channel is important is that the Higgs signal comes entirely from it. Thus, we maximize the Higgs to background ratio in two different ways.

Furthermore, at higher energies, it will be possible to produce top-quarks in photonphoton collisions. This, when combined with other data on top-quark production from $e^{+} e^{-}$ and $p \bar{p}$ collisions, should certainly improve our knowledge of the top-quark parameters. The HOC could have a significant effect on the threshold behaviour. It is also interesting to examine the spin dependence of the HOC in this region.

In this paper we present complete analytical results for heavy quark production by both polarized and unpolarized photons. Numerical results are presented for 2-, 3- and 2+3-jet cross sections for the cases where the initial photons have total spin $J_{z}=0$ and $J_{z}= \pm 2$. For $b$-quark production, this is analyzed as a background to Higgs production. We also consider $t$-quark production for energies not too far above threshold.

The analytical results presented here are also useful in determining the production of heavy quarks in polarized photon-proton (proton-proton) collisions. This is because the process $\gamma \gamma \rightarrow Q \bar{Q}(g)$ is the Abelian (QED) part of the subprocess $\gamma g \rightarrow Q \bar{Q}(g)$ $(g g \rightarrow Q \bar{Q}(g))$, which is by far the dominant subprocess in $\gamma-p(p-p)$ collisions. 国 The non-Abelian part of $\vec{\gamma} \vec{g} \rightarrow Q \bar{Q}(g)(\vec{g} \vec{g} \rightarrow Q \bar{Q}(g))$ remains to be calculated.

\section{LEADING ORDER CROSS SECTIONS}

The contributing graphs are shown in Fig. 1. We introduce the variables (momenta as in figure)

$$
s \equiv\left(p_{1}+p_{2}\right)^{2}, \quad t \equiv T-m^{2} \equiv\left(p_{1}-p_{3}\right)^{2}-m^{2}, \quad u \equiv U-m^{2} \equiv\left(p_{2}-p_{3}\right)^{2}-m^{2}
$$




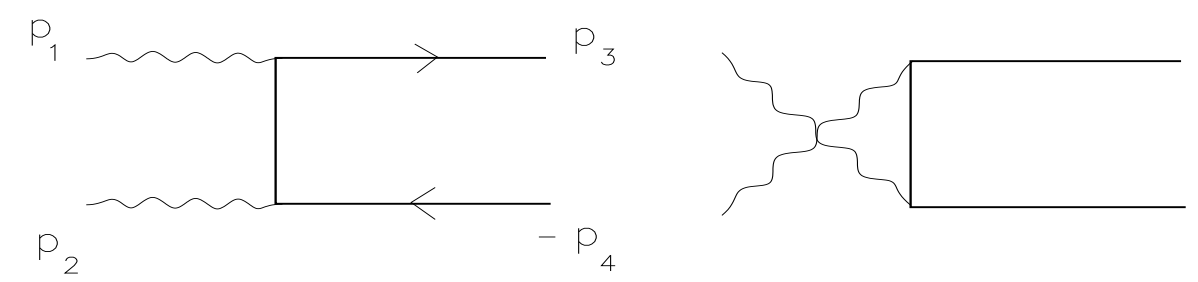

Figure 1: Lowest order contributions to $\gamma \gamma \rightarrow Q \bar{Q}$.

and

$$
s_{2} \equiv S_{2}-m^{2} \equiv\left(p_{1}+p_{2}-p_{3}\right)^{2}-m^{2}=s+t+u \text {. }
$$

where $m$ is the heavy-quark mass. Defining

$$
v \equiv 1+\frac{t}{s}, \quad w \equiv \frac{-u}{s+t}
$$

we may express

$$
t=-s(1-v), \quad u=-s v w, \quad s_{2}=s v(1-w) .
$$

The polarized and unpolarized squared amplitudes are defined, respectively, as

$$
\Delta|M|^{2} \equiv \frac{1}{2}\left(|M(+,+)|^{2}-|M(+,-)|^{2}\right), \quad|M|^{2} \equiv \frac{1}{2}\left(|M(+,+)|^{2}+|M(+,-)|^{2}\right),
$$

where $M\left(\lambda_{1}, \lambda_{2}\right)$ denotes the Feynman amplitude with photons $p_{1}, p_{2}$ having helicity $\lambda_{1}$, $\lambda_{2}$ respectively. The same holds for the cross sections.

For the examples considered in this paper, it is of interest to calculate (numerically) the cross sections for a specific helicity state, $\sigma\left(\lambda_{1}, \lambda_{2}\right)$. We present analytical results for the polarized and unpolarized cross sections $\Delta \sigma, \sigma$. From (5) we can obtain the desired cross sections via

$$
\sigma(+,+)=\sigma+\Delta \sigma, \quad \sigma(+,-)=\sigma-\Delta \sigma .
$$

Defining

$$
K(\varepsilon) \equiv \frac{m^{-2 \varepsilon}}{s} \frac{\pi(4 \pi)^{-2+\varepsilon}}{\Gamma(1-\varepsilon)}\left(\frac{t u-s m^{2}}{s m^{2}}\right)^{-\varepsilon}
$$

we may express the $n(=4-2 \varepsilon)$-dimensional 2-body phase space as

$$
[\Delta] \frac{d \sigma_{2 \rightarrow 2}}{d v d w}=K(\varepsilon)\left[(2 m)^{2}[\Delta]|M|_{2 \rightarrow 2}^{2}\right] \delta(1-w) .
$$

It will become necessary to work in $n$ dimensions when we determine the HOC (see next section for details).

The resulting leading-order ( $\mathrm{LO})$ cross sections are, in $n$ dimensions,

$$
\begin{aligned}
\Delta \frac{d \sigma_{\mathrm{LO}}}{d v d w} & =32 \pi^{2} K(\varepsilon) \delta(1-w) N_{C} \alpha^{2} e_{Q}^{4} \mu^{4 \varepsilon}\left\{-\frac{t^{2}+u^{2}}{t u}+2 \frac{s m^{2}}{t u}\left(\frac{s^{2}}{t u}-2\right)\right\} \\
\frac{d \sigma_{\mathrm{LO}}}{d v d w} & =32 \pi^{2} K(\varepsilon) \delta(1-w) N_{C} \alpha^{2} e_{Q}^{4} \mu^{4 \varepsilon}\left\{\frac{t^{2}+u^{2}}{t u}+4 \frac{s m^{2}}{t u}-4\left(\frac{s m^{2}}{t u}\right)^{2}\right\}
\end{aligned}
$$




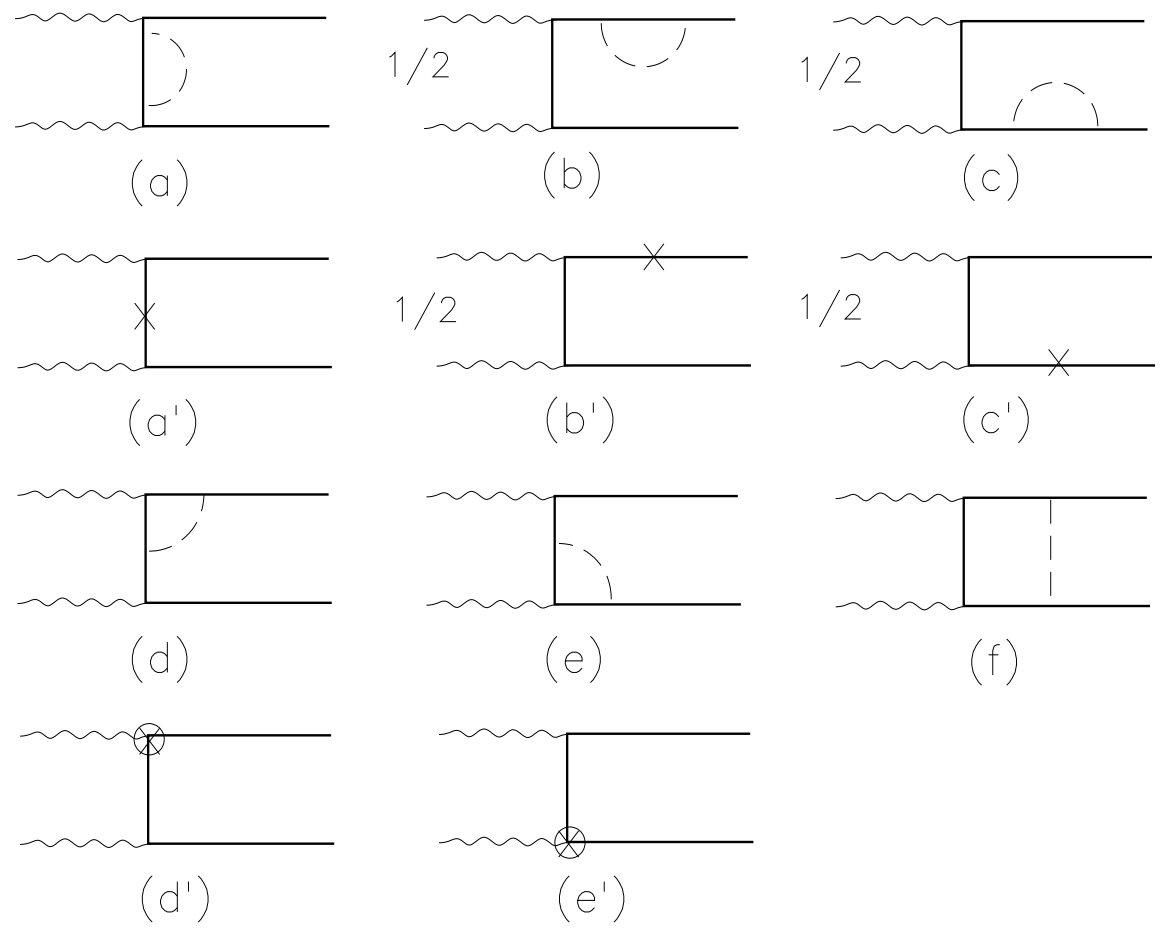

Figure 2: Loop graphs for $\gamma \gamma \rightarrow Q \bar{Q}$. (a) - (c) self-energy diagrams; $\left(\mathbf{a}^{\prime}\right)-\left(\mathbf{c}^{\prime}\right)$ mass counterterm diagrams corresponding to the graphs (a)-(c); (d),(e) vertex diagrams; $\left(\mathbf{d}^{\prime}\right),\left(\mathbf{e}^{\prime}\right)$ dimensional reduction counterterm diagrams corresponding to graphs (d),(e); (f) box diagram.

where $N_{C}(=3)$ is the number of quark colors and $e_{Q}$ is the fractional charge of the heavy quark. Making use of (4⿴囗十), (6) we see explicity that $d \sigma_{\mathrm{LO}}(+,+) / d v d w$ is suppressed by order $m^{2} / s$.

\section{LOOP CONTRIBUTIONS}

The loop contributions arise from the diagrams of Fig. 2 and their $p_{1} \leftrightarrow p_{2}$ interchange. These diagrams contain both ultraviolet and infrared singularities. To regularize them, we use dimensional reduction 6 , where the momenta are in $n$ dimensions and everything else is in four dimensions. This facilitates the handling of the Levi-Civita tensor $\varepsilon^{\mu \nu \lambda \rho}$. As we will show below, the analytical expressions for the cross sections are regularization scheme independent once all the contributions (including the gluonic bremsstrahlung) are added. Throughout, we work in the Feynman gauge.

The heavy-quark mass and wave function renormalizations are performed on-shell. The self-energy graphs are shown in Figs. 2 (a)-(c) and the corresponding mass counterterm diagrams in Figs. $2\left(\mathrm{a}^{\prime}\right)-\left(\mathrm{c}^{\prime}\right)$. The factor $1 / 2$ multiplying $(\mathrm{b})-\left(\mathrm{c}^{\prime}\right)$ comes from wave func- 
tion renormalization. The bare mass and wave function are determined in terms of the renormalized ones via

$$
m_{0}=Z_{m} m_{r}, \quad \Psi_{0}=Z_{2}^{1 / 2} \Psi_{r},
$$

where $Z_{m}$ and $Z_{2}$ are the mass and wave function renormalization constants. Define

$$
C_{\varepsilon} \equiv \frac{\Gamma(1+\varepsilon)}{(4 \pi)^{2}}\left(\frac{4 \pi \mu^{2}}{m^{2}}\right)^{\varepsilon}
$$

where $\mu$ is an arbitrary mass scale which enters via the coupling in $n$ dimensions: $g \rightarrow g \mu^{\varepsilon}$. In dimensional reduction we find, to order $g^{2}$,

$$
Z_{m}=1-3 g^{2} C_{\varepsilon} C_{F}\left(\frac{1}{\varepsilon^{\prime}}+\frac{5}{3}\right), \quad Z_{2}=1-g^{2} C_{\varepsilon} C_{F}\left(\frac{1}{\varepsilon^{\prime}}+5+\frac{2}{\varepsilon}\right)
$$

with $C_{F}=4 / 3$. We use $1 / \varepsilon^{\prime}$ to indicate which terms are of ultraviolet origin.

In dimensional reduction we must add to the vertex diagrams of Figs. 2 (d), (e) appropriate counterterms $\left(d^{\prime}\right),\left(e^{\prime}\right)$ in order to satisfy the Ward identity

$$
Z_{1}=Z_{2}
$$

between vertex and self-energy graphs, with $Z_{1}$ denoting the vertex renormalization constant. The Feynman rule for this vertex counterterm is found to be (in $n$ dimensions)

$$
\gamma^{\mu} \rightarrow \frac{-g^{2}}{(4 \pi)^{2}} C_{F} \gamma_{\varepsilon}^{\mu} \frac{1}{\varepsilon^{\prime}}
$$

with

$$
\gamma_{\varepsilon}^{\mu}=\left(g^{\mu \nu}-g_{n}^{\mu \nu}\right) \gamma_{\nu}, \quad g_{n}^{\mu \nu} g_{\mu \nu}^{n}=g_{n}^{\mu \nu} g_{\mu \nu}=n
$$

here $g_{n}^{\mu \nu}$ represents the $n$-dimensional metric tensor with, formally, $n<4$.

When all the contributions to the physical cross section (including gluonic bremsstrahlung) are added, the result is free of infrared divergences as there are no collinear singularities here. Thus the only scheme dependent part might come from the vertex and self-energy graphs. Having satisfied the Ward identity (13) though, means that the scheme dependent part of the corrections cancels between vertex and self-energy graphs. This was explicitly verified by calculating the vertex and self-energy graphs in dimensional regularization. We also checked explicitly that there are no differences between reduction and regularization arising from any other contributions. More specifically, to obtain the dimensional regularization result for any particular contribution given in this paper, simply replace the LO term by the corresponding LO term from dimensional regularization. When all the contributions are added, the scheme dependent part of the LO term cancels along with the $1 / \varepsilon$ infrared divergence multiplying it. Hence, the absence of collinear divergences or vacuum polarization graphs leads to scheme independence.

As was stated in Refs. 8 and 9, a counterterm like (14) was used to remove an unphysical term. General one-loop counterterms have been developed 10 to convert unpolarized 
dimensional reduction results into the corresponding dimensional regularization results for the purely massless case. Also, certain equivalences between dimensional reduction and dimensional regularization have been noted.11 In the present case however, satisfaction of (13) is sufficient to ensure scheme independence.

Adding the contributions of Figs. 2 (a)-( $\mathrm{e}^{\prime}$ ) (and the $t \leftrightarrow u$ interchange) resulted in the ultraviolet finite vertex plus self-energy cross section

$$
\begin{aligned}
& \frac{d \sigma_{\mathrm{vse}}}{d v d w}=-\frac{16}{\varepsilon} \pi \alpha_{s} C_{F} C_{\varepsilon} \frac{d \sigma_{\mathrm{LO}}}{d v d w}+\delta(1-w) \frac{C K(0)}{(4 \pi)^{2}}\left\{2 A _ { 1 } \left[4\left(\xi(2)-\mathrm{Li}_{2}\left(\frac{T}{m^{2}}\right)\right)\left(1+3 \frac{m^{2}}{t}\right)\right.\right. \\
& \left.\left.-\ln \left(-\frac{t}{m^{2}}\right)\left(8-6 \frac{t}{T}-\frac{t^{2}}{T^{2}}\right)-2-\frac{t}{T}\right]+A_{2} \ln \left(-\frac{t}{m^{2}}\right)+A_{3}\left(\operatorname{Li}_{2}\left(\frac{T}{m^{2}}\right)-\xi(2)\right)+A_{4}+(t \leftrightarrow u)\right\}
\end{aligned}
$$

where

$$
C \equiv(4 \pi)^{3} C_{F} N_{C} \alpha_{s} \alpha^{2} e_{Q}^{4} \mu^{6 \varepsilon} .
$$

The corresponding polarized cross section, $\Delta d \sigma_{\mathrm{vse}} / d v d w$ can be obtained by replacing the $A_{i}$ and $d \sigma_{\mathrm{LO}} / d v d w$ in (16) by $\Delta A_{i}$ and $\Delta d \sigma_{\mathrm{LO}} / d v d w$, respectively. The $[\Delta] A_{i}$ are given in Appendix B. We will use this notation throughout. We note the term $\sim 1 / \varepsilon$ in (16) representing an infrared divergence. Also, note that $[\Delta] A_{1}$ is proportional to the LO squared amplitude without the $t \leftrightarrow u$ interchange (see Appendix B, Eq. B3).

Since $[\Delta] d \sigma_{\mathrm{LO}} / d v d w$ is in general regularization scheme dependent to $\mathcal{O}(\varepsilon)$ (working in $n$ dimensions), we see explicitly that truly scheme independent cross sections will result only when all contributions are added and all infrared divergences are cancelled.

In order to evaluate the box graph of Fig. 2 (f), we must reduce the resulting tensor integrals to scalar ones (conveniently listed in Ref. 2) using projective tensor techniques. 12 The tensor integrals have the general form

$$
\begin{aligned}
& D^{0, \mu, \mu \nu, \mu \nu \lambda}\left(k_{1}, k_{2}, k_{3}, m_{1}, m_{2}, m_{3}, m_{4}\right) \equiv \\
& \quad \mu^{2 \varepsilon} \int \frac{d^{n} q}{(2 \pi)^{n}} \frac{1, q^{\mu}, q^{\mu} q^{\nu}, q^{\mu} q^{\nu} q^{\lambda}}{\left(q-m_{1}\right)^{2}\left[\left(q+k_{1}\right)^{2}-m_{2}^{2}\right]\left[\left(q+k_{1}+k_{2}\right)^{2}-m_{3}^{2}\right]\left[\left(q+k_{1}+k_{2}+k_{3}\right)^{2}-m_{4}^{2}\right]}
\end{aligned}
$$

where the $k_{i}$ are general momenta. As an example, the vector box integral we encounter has the decomposition

$$
D^{\mu}\left(p_{4},-p_{2},-p_{1}, 0, m, m, m\right)=p_{4}^{\mu} D_{11}-p_{2}^{\mu} D_{12}-p_{1}^{\mu} D_{13} .
$$

In general, the scalar coefficients $D_{i j}$ are not independent. This simplifies somewhat the calculation. Noting that

$$
D^{\mu}\left(p_{4},-p_{2},-p_{1}, 0, m, m, m\right)=-D^{\mu}\left(p_{3},-p_{1},-p_{2}, 0, m, m, m\right),
$$

we obtain

$$
D_{12}=D_{11}-D_{13},
$$

since the $D_{i j}$ in both integrals are the same, due to the fact that they are scalars. Using the same approach, we reduce the number of independent $D_{i j}$ from seven to five in $D^{\mu \nu}$ 
and from thirteen to eight in $D^{\mu \nu \lambda}$. This method was quite helpful in keeping the very large intermediate expressions as short as possible.

Adding the contribution of Fig. 2 (f) and the $t \leftrightarrow u$ interchange gives the virtual box cross section

$$
\begin{aligned}
\frac{d \sigma_{\mathrm{box}}}{d v d w} & =16 \pi \alpha_{s} C_{F} C_{\varepsilon} \frac{d \sigma_{\mathrm{LO}}}{d v d w} \frac{2 m^{2}-s}{s \beta}\left\{2 \ln (x)\left[\frac{1}{2 \varepsilon}-\ln (\beta)\right]+2 \mathrm{Li}_{2}(-x)-2 \mathrm{Li}_{2}(x)-3 \xi(2)\right\} \\
& +\delta(1-w) \frac{C K(0)}{(4 \pi)^{2}}\left\{-8 B_{1} \frac{2 m^{2}-s}{s \beta} \ln (x) \ln \left(-t / m^{2}\right)+2 \frac{B_{2}}{\beta}[\ln (x)(4 \ln (1+x)-\ln (x)\right. \\
& \left.\left.-4 \ln \left(-t / m^{2}\right)\right)+4 \mathrm{Li}_{2}(-x)+2 \xi(2)\right]+2 B_{3} \ln ^{2}(x)+4 \frac{B_{4}}{\beta} \ln (x)+4 B_{5} \ln \left(-t / m^{2}\right) \\
& \left.+8 B_{6} \operatorname{Li}_{2}\left(T / m^{2}\right)+4 B_{7} \xi(2)+4 B_{8}+(t \leftrightarrow u)\right\}
\end{aligned}
$$

where

$$
\xi(2)=\frac{\pi^{2}}{6}, \quad \beta \equiv \sqrt{1-4 m^{2} / s}, \quad x \equiv \frac{1-\beta}{1+\beta} .
$$

The $[\Delta] B_{i}$ are given in Appendix B. We see again the infrared divergence $\sim 1 / \varepsilon$.

Independent calculations were performed using FORM 13 and REDUCE 14 . The latter proved useful in factoring the expressions and cancelling powers in the denominators.

\section{GLUONIC BREMSSTRAHLUNG CONTRIBUTIONS}

The bremsstrahlung diagrams are shown in Fig. 3. Squaring these diagrams (plus their $p_{1} \leftrightarrow p_{2}$ interchange), we obtain the $2 \rightarrow 3$ particle squared amplitude

$$
\begin{aligned}
(2 m)^{2}|M|_{2 \rightarrow 3}^{2}= & \\
& C\left[\frac{\tilde{e}_{1}}{s_{2}^{2}}+e_{2} p_{2} \cdot k+\frac{e_{3}}{p_{2} \cdot p_{4}}+\frac{e_{4}}{p_{2} \cdot p_{4}^{2}}+e_{5} \frac{p_{2} \cdot k}{p_{1} \cdot p_{4}^{2}}+\frac{\tilde{e}_{6}}{s_{2} p_{3} \cdot k}+\frac{\tilde{e}_{7}}{p_{3} \cdot k^{2}}\right. \\
+ & \frac{e_{8}}{p_{1} \cdot p_{4} p_{2} \cdot p_{4}}+e_{9} \frac{p_{2} \cdot k^{2}}{p_{1} \cdot p_{4}}+e_{10} \frac{p_{2} \cdot k}{p_{1} \cdot p_{4}}+\frac{\tilde{e}_{11} / s_{2}}{p_{2} \cdot p_{4} p_{3} \cdot k}+\frac{\tilde{e}_{12}}{p_{2} \cdot p_{4} p_{3} \cdot k^{2}} \\
+ & \left.\frac{e_{13}}{p_{2} \cdot p_{4}^{2} p_{3} \cdot k}+\frac{\tilde{e}_{14}}{p_{2} \cdot p_{4}^{2} p_{3} \cdot k^{2}}+e_{15} \frac{p_{2} \cdot k^{2}}{p_{3} \cdot k}+e_{16} \frac{p_{2} \cdot k}{p_{3} \cdot k}\right]+\left(p_{1} \leftrightarrow p_{2}, t \leftrightarrow u\right)
\end{aligned}
$$

As before, we may obtain $\Delta|M|_{2 \rightarrow 3}^{2}$ by replacing the $e_{i}$ in (24) by $\Delta e_{i}$. The $[\Delta] e_{i}$ are given in Appendix B. Again, independent calculations were performed using FORM and REDUCE. The former proved useful in partial fractioning and other reductions of the dot-products.

To obtain the total bremsstrahlung contribution to $[\Delta] d \sigma / d v d w$, we perform the phasespace integrals in the frame where $p_{4}$ and $k$ are back-to-back. We find (in agreement with Ref. 2) for the $2 \rightarrow 3$ phase space

$$
[\Delta] \frac{d \sigma_{\mathrm{Br}}}{d v d w}=K(\varepsilon) \frac{C_{\varepsilon}}{\mu^{2 \varepsilon}} \tilde{f}(\varepsilon) \int d \Omega(2 m)^{2}[\Delta]|M|_{2 \rightarrow 3}^{2}
$$


where

$$
\tilde{f}(\varepsilon) \equiv \frac{\left(m^{2}\right)^{1-\varepsilon}}{S_{2}^{1-\varepsilon}} \frac{s v}{2 \pi}\left(\frac{s v}{m^{2}}\right)^{1-2 \varepsilon}(1-w)^{1-2 \varepsilon}
$$

and

$$
\int d \Omega \equiv \int_{0}^{\pi} d \theta_{1} \sin ^{1-2 \varepsilon} \theta_{1} \int_{0}^{\pi} d \theta_{2} \sin ^{-2 \varepsilon} \theta_{2}
$$

The gluon angles $\theta_{1}$ and $\theta_{2}$ are defined in Appendix $\mathrm{A}$ along with all the momenta.

We first evaluate all the phase space integrals in four dimensions since, for $w \neq 1$, all the integrals are finite. For $w=1$, the terms in (24) with coefficients $\tilde{e}_{i}$ are singular through the relation 15

$$
(1-w)^{-1-2 \varepsilon}=-\frac{1}{2 \varepsilon} \delta(1-w)+\frac{1}{(1-w)_{+}}+\mathcal{O}(\varepsilon)
$$

where the function $1 /(1-w)_{+}$is defined through

$$
\int_{w_{1}}^{1} d w \frac{f(w)}{(1-w)_{+}}=\int_{w_{1}}^{1} d w \frac{f(w)-f(1)}{(1-w)}+f(1) \ln \left(1-w_{1}\right) .
$$

This means that, for these terms, the integrals must also be evaluated in $n$ dimensions in the limit $w \rightarrow 1$, keeping their $\mathcal{O}(\varepsilon)$ part. The resulting integrals are straightforward.

The final result is, with $\bar{y} \equiv \sqrt{(t+u)^{2}-4 m^{2} s}$,

$$
\begin{aligned}
& \frac{d \sigma_{\mathrm{Br}}}{d v d w}= \\
& \quad \frac{C K(0)}{(4 \pi)^{2}} 2 \pi \tilde{f}(0)\left\{\frac{s_{2}(s+u)}{4 S_{2}} e_{2}+\frac{2 S_{2}}{s_{2}(s+u)} \ln \frac{S_{2}}{m^{2}} e_{3}+\frac{4 S_{2}}{m^{2}(s+u)^{2}} e_{4}\right. \\
& \left.+\quad e_{5} I_{5}+e_{8} I_{8}+e_{9} I_{9}+e_{10} I_{10}+e_{13} I_{13}+e_{15} I_{15}+e_{16} I_{16}+(t \leftrightarrow u)\right\} \\
& +\quad \frac{1}{(1-w)_{+}} \frac{C K(0)}{(4 \pi)^{2}} \frac{1}{S_{2}}\left\{\tilde{e}_{1}+\frac{2 S_{2}}{\bar{y}} \ln \frac{T+U-\bar{y}}{T+U+\bar{y}} \tilde{e}_{6}+4 \frac{S_{2}}{m^{2}} \tilde{e}_{7}+s_{2} I_{11} \tilde{e}_{11}\right. \\
& \left.+\quad s_{2}^{2} I_{12} \tilde{e}_{12}+s_{2}^{2} I_{14} \tilde{e}_{14}+(t \leftrightarrow u)\right\}+8 \pi \alpha_{s} C_{F} C_{\varepsilon} \frac{d \sigma_{\mathrm{LO}}}{d v d w} \frac{1}{s \beta}\left\{\left(2 m^{2}-s\right)\right. \\
& \left.\times \quad\left[2 \ln x\left(2 \ln \frac{s v}{m^{2}}-\frac{1}{\varepsilon}\right)-1-2\left(\operatorname{Li}_{2}\left(\frac{-4 \beta}{(1-\beta)^{2}}\right)+\ln ^{2} x\right)\right]+2 s \beta\left[1-2 \ln \frac{s v}{m^{2}}+\frac{1}{\varepsilon}\right]\right\}
\end{aligned}
$$

where the integrals $I_{i}$ are given in Appendix C.

\section{PHYSICAL CROSS SECTIONS}

We may obtain the $2+3$-jet cross section by adding (9), (16), (22), and (30):

$$
[\Delta] \frac{d \sigma_{2+3}}{d v d w}=[\Delta] \frac{d \sigma_{\mathrm{LO}}}{d v d w}+[\Delta] \frac{d \sigma_{\mathrm{vse}}}{d v d w}+[\Delta] \frac{d \sigma_{\mathrm{box}}}{d v d w}+[\Delta] \frac{d \sigma_{\mathrm{Br}}}{d v d w}
$$



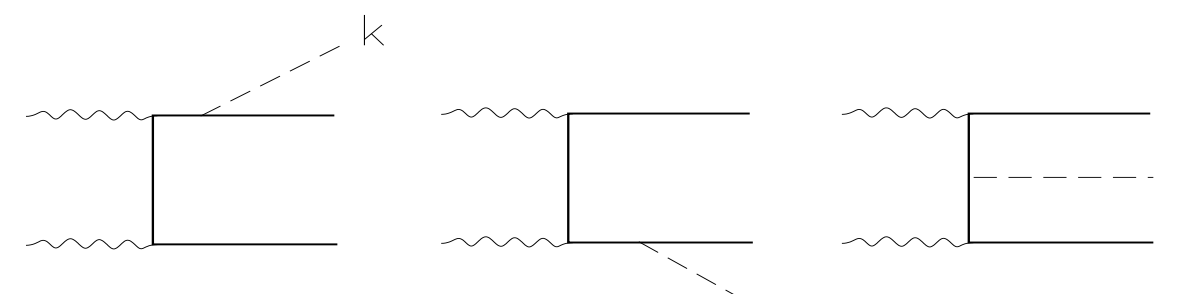

Figure 3: Gluonic Bremsstrahlung graphs for $\gamma \gamma \rightarrow Q \bar{Q} g$.

We notice the cancellation of all the $1 / \varepsilon$ infrared divergences, leading to a finite, scheme independent result.

At this point it is useful to note that for $s \gg 4 m^{2}$, the LO cross sections (9) are large in the forward and backward directions. Since jets going down the beam pipe are difficult to measure experimentally, angular cuts are necessary for $b \bar{b}$ production well above threshold. At the same time, we reduce the $b \bar{b}$ background to the Higgs signal. This also helps eliminate resolved photon contributions where the partons within the photon participate, as opposed to the direct contributions, which we present, where the photon is structureless. This is discussed at the end of this section.

Let $\theta_{3}$ denote the angle between $p_{3}$ and $p_{1}$ in the $\gamma \gamma$ c.m. Then the integrated $2+3$-jet cross section, with the constraint $\left|\cos \theta_{3}\right|<\cos \theta_{c}$, for some $\theta_{c}$, is given by

$$
[\Delta] \sigma_{2+3}(s)=\int_{v_{1}}^{v_{2}} d v \int_{w_{1}}^{1} d w \Theta\left(\cos ^{2} \theta_{c}-\cos ^{2} \theta_{3}\right)[\Delta] \frac{d \sigma_{2+3}}{d v d w}
$$

where

$$
v_{1}=\frac{1}{2}(1-\beta), \quad v_{2}=\frac{1}{2}(1+\beta), \quad w_{1}(v)=\frac{m^{2}}{s} \frac{1}{v(1-v)}
$$

and

$$
\cos \theta_{3}=\frac{-(1-v-v w)}{\sqrt{(1-v+v w)^{2}-4 m^{2} / s}} .
$$

Alternatively, we may convert to $d \sigma / d \cos \theta_{3} d w$ and integrate directly over $\theta_{3}$ and $w$.

The integrated 3 -jet cross section is given by

$$
\begin{aligned}
{[\Delta] \sigma_{3}(s) } & =\frac{K(0)}{(4 \pi)^{2}} \int_{v_{1}}^{v_{2}} d v \int_{w_{1}}^{1} d w \Theta\left(\cos ^{2} \theta_{c}-\cos ^{2} \theta_{3}\right) \tilde{f}(0) \int d \Omega(2 m)^{2}[\Delta]|M|_{2 \rightarrow 3}^{2} \\
& \times \Theta\left(\left(p_{3}+k\right)^{2}-y_{\text {cut }} s\right) \Theta\left(\left(p_{4}+k\right)^{2}-y_{\text {cut }} s\right) \\
& =\frac{K(0)}{(4 \pi)^{2}} \int_{v_{1}}^{v_{2}} d v \int_{w_{1}}^{w_{2}} d w \Theta\left(\cos ^{2} \theta_{c}-\cos ^{2} \theta_{3}\right) \tilde{f}(0) \int d \Omega(2 m)^{2}[\Delta]|M|_{2 \rightarrow 3}^{2} \\
& \times \Theta\left(\left(p_{3}+k\right)^{2}-y_{\text {cut }} s\right) ; \quad w_{2}=1-\frac{\left(y_{\text {cut }}-m^{2} / s\right)}{v} .
\end{aligned}
$$

The angular integral is given by (27) with $\varepsilon=0$. The dot-products involved may be explicitly expressed as functions of $v, w, \theta_{1}$ and $\theta_{2}$ using the parametrizations of Appendix 
A and Eqs. (4). We have imposed the constraints, $\left(p_{3}+k\right)^{2}>y_{\text {cut }} s$ and $\left(p_{4}+k\right)^{2}>y_{\text {cut }} s$. With a suitable choice of $y_{\text {cut }}$ we may simultaneously cut out events with 2-jet topology and avoid the soft divergence. We effectively eliminate the soft and collinear gluons from the 3 -jet cross section, with the degree of softness and collinearity being specified by $y_{\text {cut }} s$.

The desired 2-jet cross section is obtained by the difference

$$
[\Delta] \sigma_{2}(s)=[\Delta] \sigma_{2+3}(s)-[\Delta] \sigma_{3}(s)
$$

Since $\sigma_{2+3}$ and $\sigma_{3}$ are both infrared finite and separately observable quantities, this serves as a reliable and unambiguous method for defining $\sigma_{2}$.

In discussing the numerical results, it will be convenient to split $[\Delta] \sigma_{2+3}$ as follows,

$$
[\Delta] \sigma_{2+3}=[\Delta] \sigma_{\mathrm{LO}}+[\Delta] \sigma_{\mathrm{S}}+[\Delta] \sigma_{\mathrm{H}}
$$

where $[\Delta] \sigma_{\mathrm{S}}$ represents the contribution to the HOC coming from terms proportional to $\delta(1-w)$ and $1 /(1-w)_{+}$, and $[\Delta] \sigma_{\mathrm{H}}$ represents the rest. In usual terminology, $[\Delta] \sigma_{\mathrm{S}}$ represents virtual and soft contributions whereas $[\Delta] \sigma_{\mathrm{H}}$ represents hard radiation.

So far we have only considered direct contributions, i.e. no resolved photon contributions. The reason is the following. Well above the $Q \bar{Q}$ threshold, $\sigma_{2+3}$ and $\sigma_{3}$ will certainly receive sizable resolved photon contributions. Now, resolved photon events are generally accompanied by a jet making small angles with respect to the beam axis. For the 2-jet cross section (which is of physical interest), experiment can reject resolved photon events (and other unwanted events) as being those for which the observed jets have total energy measurably lower than $\sqrt{s}$. This is because, due to the angular cuts, experiment will not observe the jet making small angles. Hence, there will be missing energy. Of course, we are assuming a rather well defined initial photon energy, which may be experimentally difficult.

For top-quark production, not too far above threshold, the resolved contributions will be negligible in all the cross sections. This is because the dominant resolved contribution comes from $g \gamma \rightarrow Q \bar{Q}$, where the gluon originates from one of the initial photons, having a fraction $x$ of its momentum. Near threshold, the gluon will have to carry a large fraction of the photon's momentum; and for $x \rightarrow 1$, the gluon distribution in the photon is highly suppressed. As well, 3-jet states arising from hard gluonic radiation will be suppressed due to the restricted phase space. The (near) absence of resolved contributions and the nonsuppression of the $J_{z}=0$ cross section for $2 \rightarrow 2$ kinematics, not too far above threshold, implies that we needn't worry about whether the events are 2- or 3-jet (even though 3-jet events are either very seldom or none, depending on $s$ ).

\section{NUMERICAL RESULTS}

Here we present numerical results for $b$ - and $t$-quark production in next-to-leading order. Throughout, we evaluate $\alpha_{s}\left(Q^{2}\right)$ (2-loop) with $Q^{2}=s, \Lambda=0.2 \mathrm{GeV}$ and the number of 

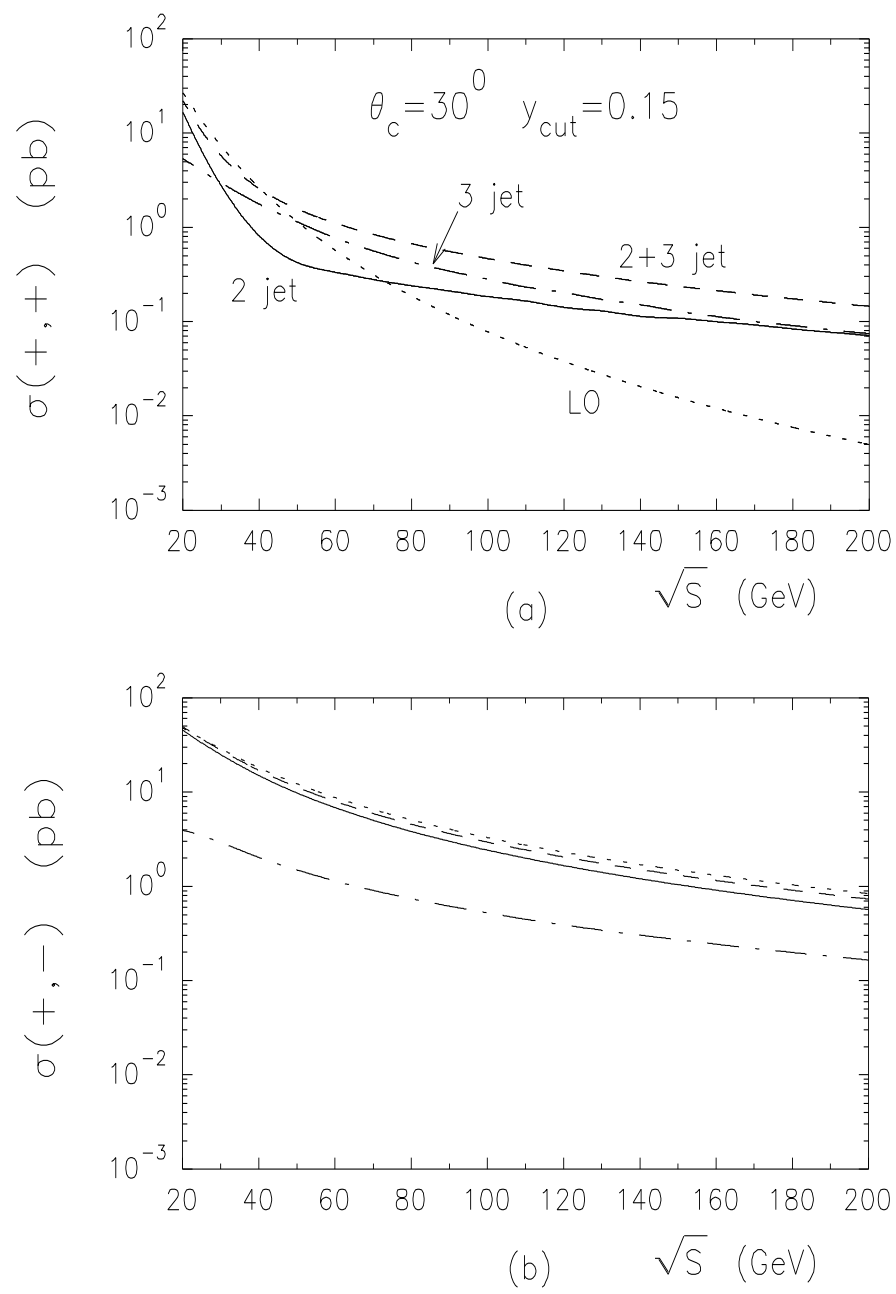

Figure 4: Cross sections for $\gamma \gamma \rightarrow b \bar{b}(g): \sigma_{\mathrm{LO}}$ (dotted line), $\sigma_{2+3}$ (dashed), $\sigma_{3}$ (dash-dotted) and $\sigma_{2}$ (solid), with $\theta_{c}=30^{0}$ and $y_{\text {cut }}=0.15$ for $20<\sqrt{s}<200 \mathrm{GeV}$; (a) $\sigma(+,+)$; (b) $\sigma(+,-)$.

flavors taken as $N_{F}=5$ since we are well above the $b \bar{b}$ threshold. We take $m_{b}=4.7 \mathrm{GeV}$ and $m_{t}=174 \mathrm{GeV}$. 16 For 3 -jet cross sections, we use $y_{\text {cut }}=0.15$. Some justification for this choice of $y_{\text {cut }}$ is in order. Experimentally, it is useful to have a small value of $y_{\text {cut }}$ so that for the 2-jet cross section we eliminate, as much as possible, events with 3-jet topology via (36). Theoretically, there are limitations. If one chooses $y_{\text {cut }}$ too small, then the infrared divergence ruins the perturbation expansion, since the 3 -jet cross section becomes unphysically large. To control this, an all-orders resummation would be required. We find that $y_{\text {cut }}=0.15$ is the most suitable choice in light of the above considerations.

Fig. 4(a) presents $\sigma_{\mathrm{LO}}(+,+), \sigma_{2+3}(+,+), \sigma_{3}(+,+)$ and $\sigma_{2}(+,+)$ for $b$-quark production in the range $20<\sqrt{s}<200 \mathrm{GeV}$ with $\theta_{c}=30^{\circ}$. As expected, the $\mathrm{LO}$ cross section is highly suppressed for large $\sqrt{s}$, but not the 3 -jet. In fact $\sigma_{3}(+,+)$ makes a sizable contribution to $\sigma_{2+3}(+,+)$. Hence $\sigma_{2}(+,+)$ gets somewhat suppressed relative to $\sigma_{2+3}(+,+)$. For $20 \lesssim \sqrt{s} \lesssim 40 \mathrm{GeV}$ the corrections $\sigma_{2+3}-\sigma_{\mathrm{LO}}$ are seen to be slightly negative.

Fig. 4 (b) presents the same cross sections for $J_{z}= \pm 2$, i.e. $\sigma_{\mathrm{LO}}(+,-), \sigma_{2+3}(+,-)$, 

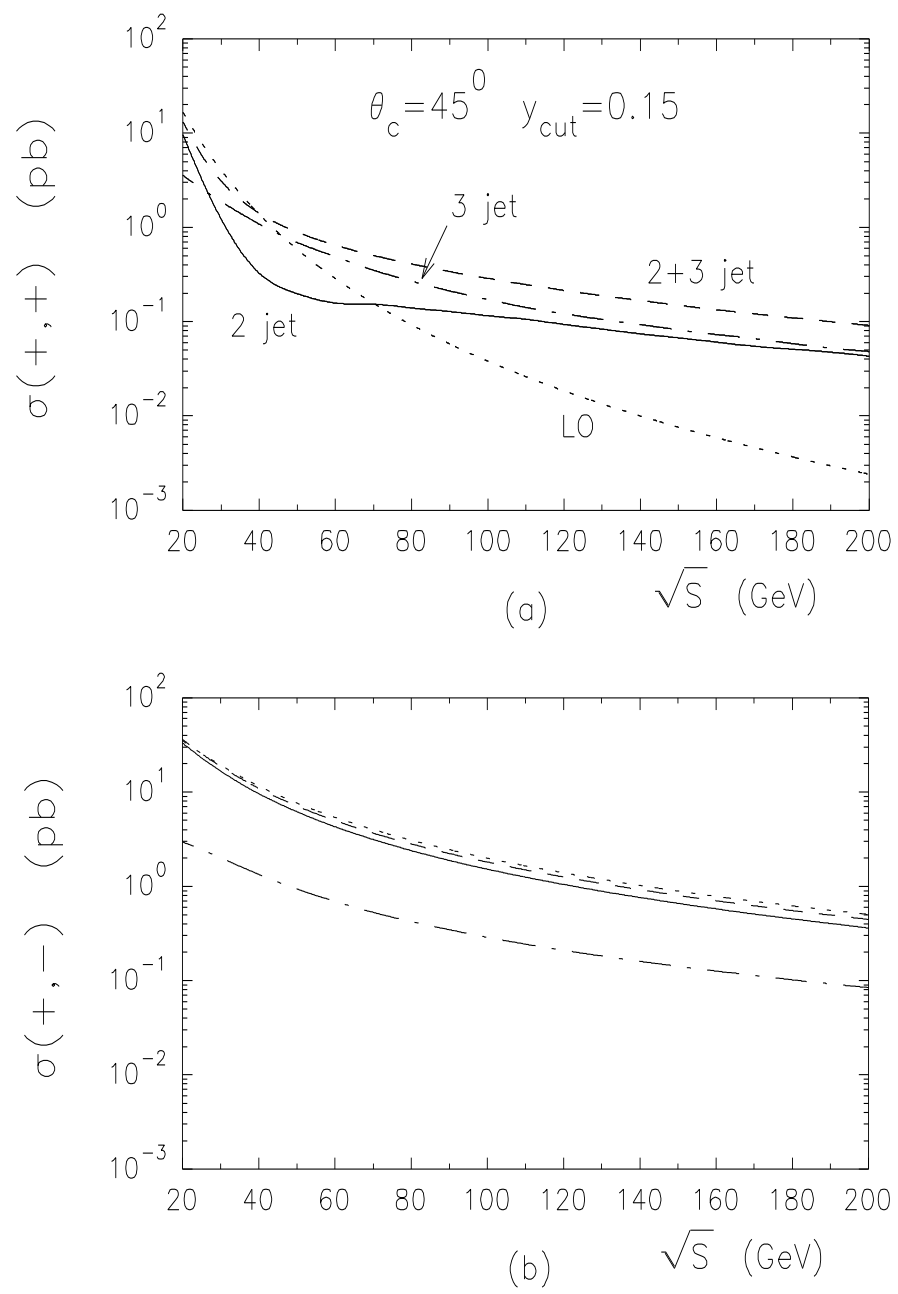

Figure 5: Same as Fig. 4, except with $\theta_{c}=45^{0}$.

$\sigma_{3}(+,-)$ and $\sigma_{2}(+,-)$. The major difference is that $\sigma_{\mathrm{LO}}(+,-)$ and $\sigma_{2+3}(+,-)$ suffer no suppression at large $\sqrt{s}$. Hence the 3 -jet contribution to $\sigma_{2+3}(+,-)$ is not so significant and $\sigma_{2}(+,-)$ remains large. We also notice, $\sigma_{2+3} \lesssim \sigma_{\text {LO }}$ throughout.

Figs. 5 (a),(b) present the same quantities as in Figs. 4 (a),(b) except with $\theta_{c}=45^{0}$. The major difference is that the cross sections are smaller everywhere and $\sigma_{2}(+,+)$ is particularly suppressed for $30 \lesssim \sqrt{s} \lesssim 60 \mathrm{GeV}$. This reflects the fact that the 2 -jet events tend to occur at smaller angles.

An interesting feature of the $\mathrm{HOC}$ arises for both $\sigma_{2+3}$ and $\Delta \sigma_{2+3}$. In both cases, $\sigma_{\mathrm{S}}$ and $\sigma_{\mathrm{H}}$ are much larger that $\sigma_{\mathrm{LO}}$, for $s \gg 4 \mathrm{~m}^{2}$. But they have opposite sign and are of almost equal magnitude, leading to large cancellations. In other words, the "virtual + soft" part conspires with the "hard" part to yield HOC which are under control.

Fig. 6 presents the 2-jet background to the Higgs decay $\gamma \gamma \rightarrow H^{*} \rightarrow b \bar{b}$. We have used the standard model Higgs cross section of Ref. 4 which takes $\theta_{c}=30^{\circ}$ and an average value of $<\lambda_{1} \lambda_{2}>=0.8$. The photons are produced by laser backscattering off electrons (positrons) 


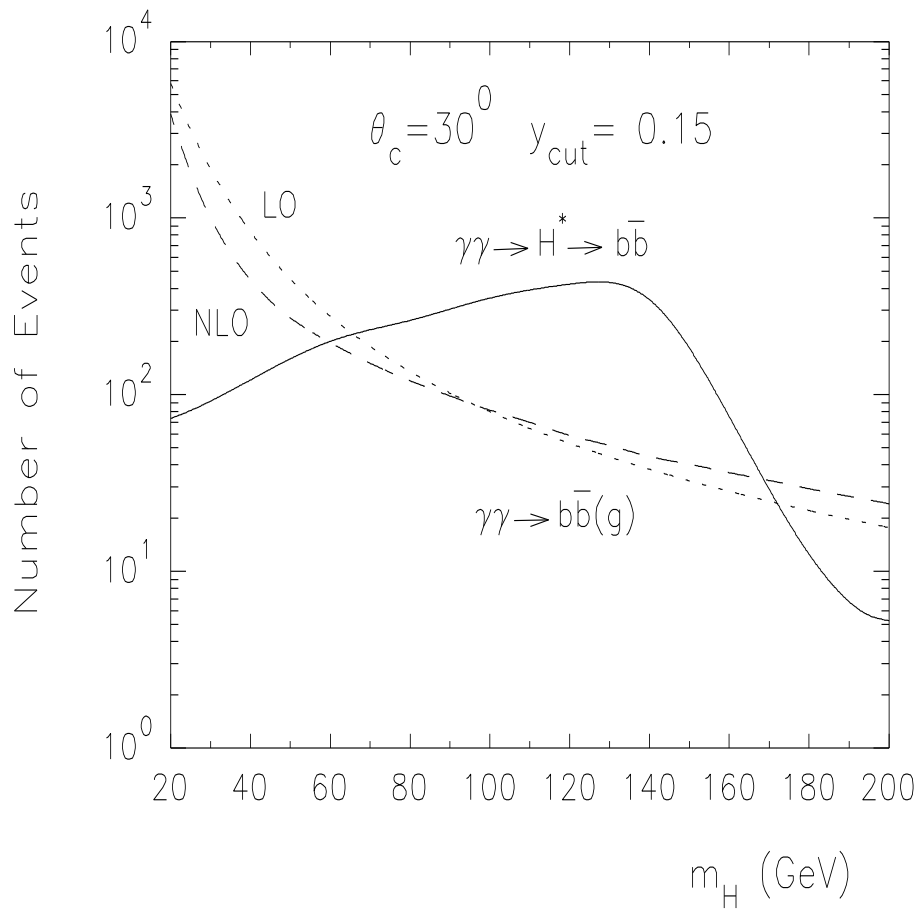

Figure 6: Two-jet $b \bar{b}$ background to standard model Higgs decay: $\gamma \gamma \rightarrow H^{*} \rightarrow b \bar{b}$ (solid line), $\sigma_{\mathrm{LO}}$ (dotted) and $\sigma_{2}$ (dashed) for $20<m_{\mathrm{H}}<200 \mathrm{GeV}$. Number of Higgs events taken from Ref. ๒. Here $\theta_{c}=30^{0},\left\langle\lambda_{1} \lambda_{2}>=0.8\right.$. The other experimental parameters are described in the text. 

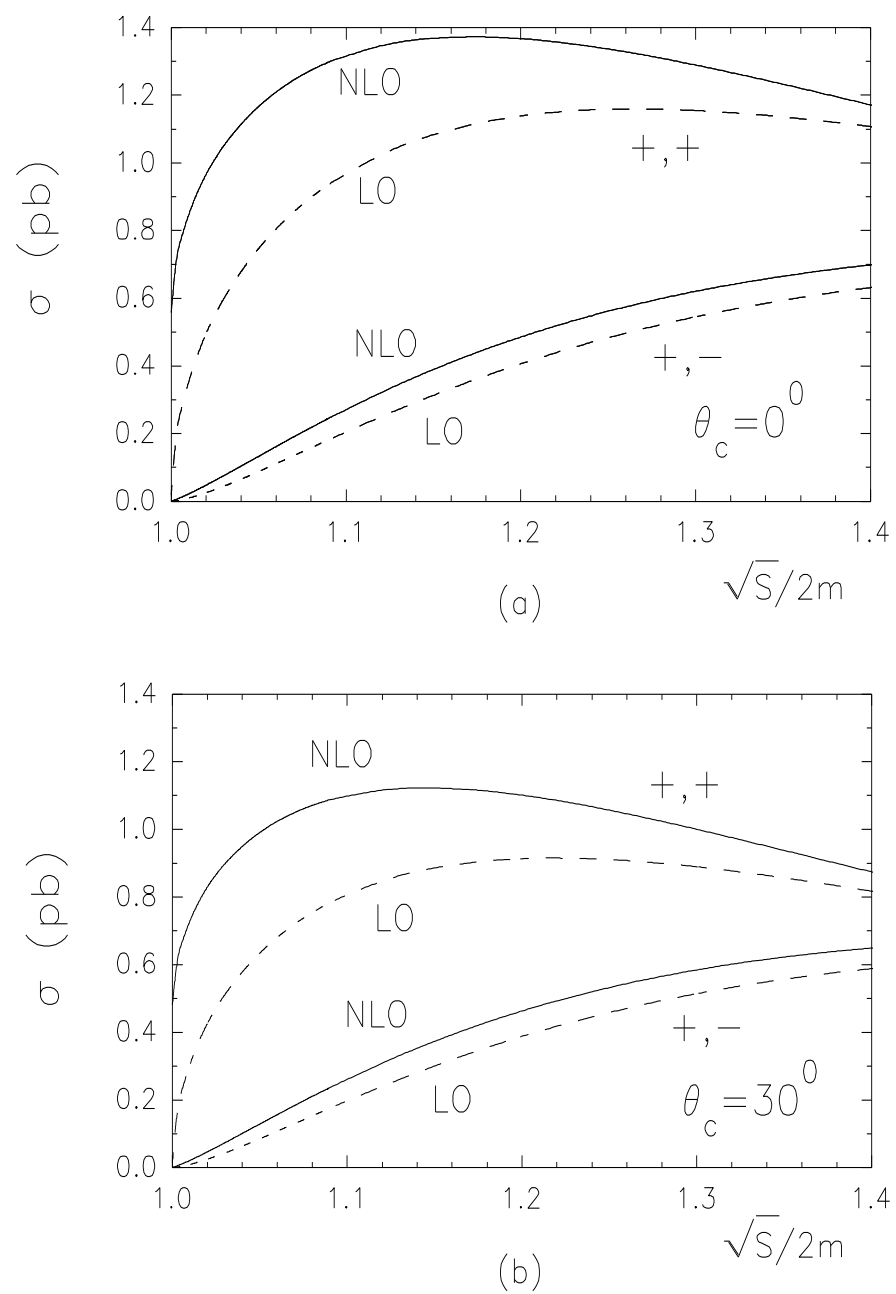

Figure 7: Cross sections for $\gamma \gamma \rightarrow t \bar{t}(g): \sigma_{\mathrm{LO}}(+,-)$ (lower dashed line), $\sigma_{2+3}(+,-)$ (lower solid), $\sigma_{\mathrm{LO}}(+,+)$ (upper dashed) and $\sigma_{2+3}(+,+)$ (upper solid) for $1<\sqrt{s} / 2 m<1.4$ : (a) $\theta_{c}=0$; (b) $\theta_{c}=30^{0}$.

at an $e^{+} e^{-}$collider with $E_{e^{+} e^{-}}=500 \mathrm{GeV}$. As well, Ref. 1 uses an effective integrated luminosity of $L_{\text {eff }}=20 \mathrm{fb}^{-1}$ and a $\gamma \gamma$ energy spread of $\Gamma_{\text {expt }}=5 \mathrm{GeV} ; \sqrt{s}=m_{\mathrm{H}} \pm \Gamma_{\text {expt }} / 2$. Using the expression of Ref. 1 for converting the $\gamma \gamma \rightarrow b \bar{b}(g)$ cross section into number of events, we obtain the LO and 2-jet curves shown in Fig. 6 .

At large $\sqrt{s}$, the increase in $\sigma_{2}(+,+)$ relative to $\sigma_{\mathrm{LO}}(+,+)$ is compensated by a decrease in $\sigma_{2}(+,-)$ relative to $\sigma_{\mathrm{LO}}(+,-)$, so that $\left.\sigma_{2}\left(<\lambda_{1} \lambda_{2}\right\rangle=0.8\right)$ doesn't change radically. In the end, the 2-jet cross section is still well below the Higgs signal for $90 \lesssim m_{\mathrm{H}} \lesssim 150 \mathrm{GeV}$. With higher degrees of polarization, we could do even better.

Fig. 7 (a) gives $\sigma_{2+3}$ and $\sigma_{\mathrm{LO}}$ for $t$-quark production in the range $1<\sqrt{s} / 2 m<1.4$ for $J_{z}=0$ and $J_{z}= \pm 2$, without angular cuts. Fig. $7(\mathrm{~b})$ is the same except with $\theta_{c}=$ $30^{\circ}$. We notice that the angular cuts do not make a big difference. This is because there is no peaked behaviour in the forward/backward directions as for $b \bar{b}$ production. As explained earlier, the (near) absence of resolved contributions makes the angular cuts less 


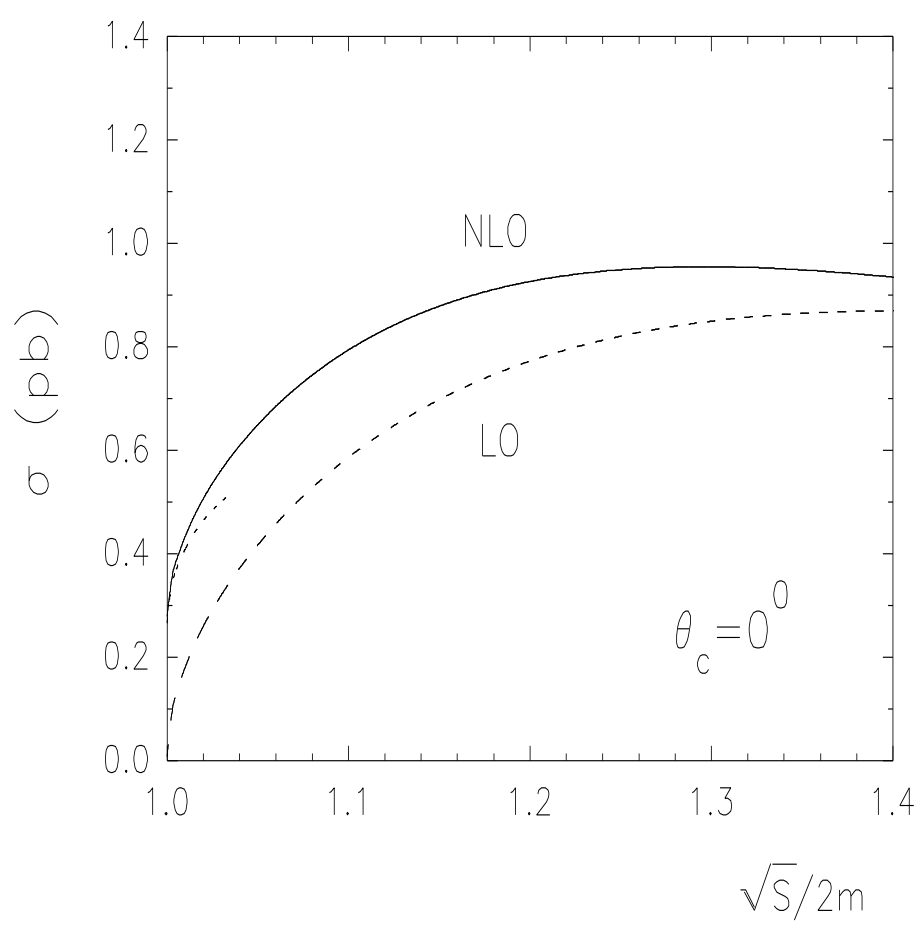

Figure 8: Unpolarized cross sections corresponding to Fig. $7(\mathrm{a}): \sigma_{2+3}$ (solid line), $\sigma_{\mathrm{LO}}$ (dashed) and the small $\beta$ approximation (dotted).

important experimentally as well. The most interesting feature of the HOC is that just above threshold, the HOC to $\sigma(+,+)$ completely dominate. There is no similar behaviour from $\sigma(+,-)$. This suggests that the $J_{z}=0$ channel is ideal for maximizing the top cross section not too far above threshold. At any rate, this drastic spin dependence of the HOC is of theoretical interest by itself and could be tested at the $b \bar{b}$ threshold as well. As the cross section is actually a function of only $\sqrt{s} / m$ (or $\beta$ ) and $\alpha_{s}\left(Q^{2}\right)$ (times an overall factor of $e_{Q}^{4} / s$ ), the only difference would be an increase in the HOC for $b \bar{b}$ relative to its corresponding LO term, due to an increase in $\alpha_{s}$. In fact, the only ambiguity in the predictions is the choice of scale $Q^{2}$ in $\alpha_{s}\left(Q^{2}\right)$. Varying $Q^{2}$ in the range $s / 4<Q^{2}<4 s$, for $\sqrt{s}=400 \mathrm{GeV}$, gives $\alpha_{s}$ in the range $0.0878<\alpha_{s}<0.104$ and a corresponding variation in the magnitude of the corrections.

Fig. 8 gives the unpolarized cross sections corresponding to Fig. 7(a). We also plot the small $\beta$ (threshold region) approximation of Ref. 3. Our results agree with this approximation just above threshold. We see that the approximation breaks down for $\sqrt{s} / 2 m \gtrsim 1.02$. As expected, we found that almost all of the correction comes from $\sigma_{\mathrm{S}}$, i.e. $\sigma_{\mathrm{H}}$ is almost negligible not too far above threshold. We found the same was true for $\Delta \sigma_{\mathrm{S}}, \Delta \sigma_{\mathrm{H}}$. 


\section{CONCLUSIONS}

We have obtained complete analytical results for the production of heavy-quark pairs by polarized and unpolarized photons in next-to-leading order. Using these expressions, we computed cross sections for $b$ - and $t$-quark production by photons having net spin $J_{z}=0, \pm 2$. From the $b \bar{b}$ cross sections, we determined the background to $\gamma \gamma \rightarrow H^{*} \rightarrow b \bar{b}$ (standard model) coming from $\gamma \gamma \rightarrow b \bar{b}(g)$ (2-jet) for $\left\langle\lambda_{1} \lambda_{2}\right\rangle=0.8$. The HOC to the $J_{z}=0$ channel were found to be large for $s \gg 4 m^{2}$. For the experimental setup considered, the background was safely below the Higgs signal (but still sizable) for $90 \lesssim m_{\mathrm{H}} \lesssim 150 \mathrm{GeV}$, even after inclusion of HOC. For $t$-quark production, not too far above threshold, the dominant contribution came from the $J_{z}=0$ channel. Just above threshold, the HOC to this channel completely dominate.

\section{ACKNOWLEDGMENTS}

We would like to thank D. Atwood, G. Jikia and O. Teryaev for discussions and J. Peralta for help in the early stages. This work was also supported by the Natural Sciences and Engineering Research Council of Canada and by the Quebec Department of Education.

\section{APPENDIX A}

Here we present the momentum parametrizations in the frame where $p_{4}$ and $k$ are back-to-back. We find

$$
\begin{aligned}
p_{1} & =\left(\omega_{1} ; 0, \cdots,|\mathbf{p}| \sin \psi,|\mathbf{p}| \cos \psi-\omega_{2}\right), \\
p_{2} & =\left(\omega_{2} ; 0, \cdots, 0, \omega_{2}\right), \\
k & =\left(\omega_{k} ; \cdots, \omega_{k} \sin \theta_{1} \cos \theta_{2}, \omega_{k} \cos \theta_{1}\right), \\
p_{4} & =\left(E_{4} ; \cdots,-\omega_{k} \sin \theta_{1} \cos \theta_{2},-\omega_{k} \cos \theta_{1}\right), \\
p_{3} & =\left(E_{3} ; 0, \cdots,|\mathbf{p}| \sin \psi,|\mathbf{p}| \cos \psi\right),
\end{aligned}
$$

where

$$
\begin{aligned}
& \omega_{1}=\frac{s+t}{2 \sqrt{S_{2}}}, \quad \omega_{2}=\frac{s+u}{2 \sqrt{S_{2}}}, \quad \omega_{k}=\frac{s_{2}}{2 \sqrt{S_{2}}}, \quad E_{4}=\frac{s_{2}+2 m^{2}}{2 \sqrt{S_{2}}} \\
& E_{3}=-\frac{T+U}{2 \sqrt{S_{2}}}, \quad|\mathbf{p}|=\frac{\bar{y}}{2 \sqrt{S_{2}}}, \quad \cos \psi=\frac{u s_{2}-s\left(t+2 m^{2}\right)}{(s+u) \bar{y}}
\end{aligned}
$$


in agreement with Ref. 2. For $p_{1}, p_{2}, p_{3}$ the dots represent zeros. For $k, p_{4}$ they represent components which depend on the remaining $n-4$ angles of $k$. Since these components do not contribute to $[\Delta]|M|_{2 \rightarrow 3}^{2}$, those angles were trivially integrated over in the phase space (25).

\section{APPENDIX B}

In this appendix we list the coefficients for the various cross sections. For $\Delta d \sigma_{\mathrm{vse}} / d v d w$ given in Eq. 16, the coefficients $\Delta A_{i}$ are

$$
\begin{aligned}
& \Delta A_{1}=2\left[-1-u / s+u^{2} / s t+m^{2} s / t u-2 m^{2} u / t^{2}\right] \\
& \Delta A_{2}=-4\left[4(6 u / t-4 s / u-t / T) m^{2} / t-4 s T / t^{2}-16 s / t+24 u / s+s / T+4 t u / s T\right] m^{2} / T \\
& \Delta A_{3}=16\left[(7 s / t+3-3 t / u) m^{4} / t^{2}+\left(4 u^{2} / s t+2 t / s-s^{2} / t u\right) m^{2} / t+t / s+u^{2} / s t\right] \\
& \Delta A_{4}=4\left[4 m^{2} u / t^{2}-3 s / t+4 u / s\right] m^{2} / T
\end{aligned}
$$

For $d \sigma_{\mathrm{vse}} / d v d w$ given in Eq. 16, the coefficients $A_{i}$ are

$$
\begin{aligned}
A_{1} & =2\left[u / t-2 m^{2} / t+s m^{2} / t u-4 m^{4} / t u-4 m^{4} / t^{2}\right] \\
A_{2} & =4\left[4\left(12 s T / t^{2}+t / T+6\right) m^{4} / u T+\left(s^{2} / u T-4 s^{2} / t u-4 s^{2} T / t^{2} u\right.\right. \\
& \left.-12 s / t-2 t / T-8) m^{2} / T-4\right] \\
A_{3} & =16\left[12 m^{6} s / t^{3} u+(s / t-14-8 t / u) m^{4} / t^{2}-(3 s / t+7+t / u) m^{2} / t+u / t\right] \\
A_{4} & =-4\left[4(2 s / t+t / T) m^{4} / t u+(t / T-4) m^{2} s^{2} / t^{2} u+2 t / T-4\right]
\end{aligned}
$$

We note that

$$
[\Delta] A_{1}+(t \leftrightarrow u)=(2 m)^{2}[\Delta]|M|_{\mathrm{LO}}^{2} /\left(N_{C} e^{4} e_{Q}^{4} \mu^{4 \varepsilon}\right) .
$$

For $\Delta d \sigma_{\text {box }} / d v d w$ given in Eq. 22, the coefficients $\Delta B_{i}$ are

$$
\begin{aligned}
\Delta B_{1} & =\Delta A_{1} \\
\Delta B_{2} & =4(s+4 t) m^{4} / s t u+2(s / u+4 u / s) m^{2} / s-s^{2} / t u-2 s / u+t / u-4 u / s \\
\Delta B_{3} & =12 m^{4} / t u+2 m^{2}(t-u) / t u-s^{2} / t u-t / u \\
\Delta B_{4} & =[8(t-u) / s-3 s / t-s / u] m^{2} / s+5 u / s-t / s \\
\Delta B_{5} & =4 s T / t u+(t / u-1) m^{2} t^{2} / s T^{2}+4\left(s^{2}-2 t^{2}\right) / s t-7 t u / s T+t^{2}(s-3 t) / s T u \\
\Delta B_{6} & =2(s / t-4) m^{4} / t u-2 m^{2}(t-u) / t u+s^{2} / t u+t / u \\
\Delta B_{7} & =4(u-4 t) m^{4} / t^{2} u+2 m^{2}(u-t) / t u+s^{2} / t u+t / u ; \quad \Delta B_{8}=\left(1+t^{2} / T s\right)(t / u-1)
\end{aligned}
$$

For $d \sigma_{\mathrm{box}} / d v d w$ given in Eq. 22, the coefficients $B_{i}$ are

$$
\begin{aligned}
& B_{1}=A_{1} ; \quad B_{2}=\left(2 m^{2}-s\right)\left[\left(2 m^{2}-u\right) / s t-2 / u\right]-2 m^{2}\left(6 m^{2}+t\right) / s u \\
& B_{3}=-4 m^{4} / t u+2 m^{2}(u-t) / t u+s^{2} / t u+t / u ; \quad B_{4}=-2 m^{2} s / t u+2 \\
& B_{5}=2\left[\left(2 m^{2}+s\right)(2 s / t+t / T)-t^{3} / T^{2}\right] / u ; \quad B_{6}=8 m^{4} / t u+2 m^{2} / u-s^{2} / t u-t / u \\
& B_{7}=-4 m^{4} / t u+2 m^{2}(t-3 u) / t u-s^{2} / t u-t / u ; \quad B_{8}=2\left(s+t^{2} / T\right) / u
\end{aligned}
$$


For $\Delta|M|_{2 \rightarrow 3}^{2}$ given in Eq. 24 and $\Delta d \sigma_{\mathrm{Br}} / d v d w$ given in Eq. 30, the coefficients $\Delta e_{i}$ are

$$
\begin{aligned}
\Delta \tilde{e}_{1} & =-16(s / u-s / t-2) m^{4} / u-4\left[s_{2}\left(2+2 s / u-t / s+u / s+2 t^{2} / s^{2}+8 t u / s^{2}\right)+2 s\right. \\
& -4 t u / s] m^{2} / u+s_{2}\left(4 s / u-4-8 t^{2} / s u-5 t / s\right) \\
\Delta e_{2} & =-4\left[2 m^{2}\left(2 / s_{2} u+2 / s_{2} t+1 / s^{2}-u / s^{2} t\right)+6 / t-t / s^{2}+u^{2} / s^{2} t\right] / u \\
\Delta e_{3} & =-2\left[8 m^{4}\left(1 / t u-1 / s_{2} u-s / s_{2} t^{2}\right)-2\left(4 s / s_{2}+s / t-1\right) m^{2} / t-3 s / u-5 s / t\right. \\
& \left.-\left(2 s^{3} / t u-s u / t+3 t+2 u+u^{2} / t\right) / s_{2}\right] \\
\Delta e_{4} & =-2\left(2 m^{2} s / t+2 s+u\right) m^{2} / t ; \quad \Delta e_{5}=0 \\
\Delta \tilde{e}_{6} & =\left[32 m^{4} / u-4 m^{2}\left(t / u+5+t^{2} / s u+5 t / s+2 t^{3} / s^{2} u+10 t^{2} / s^{2}+8 t u / s^{2}\right)\right. \\
& \left.+4 s t / u-16 t-8 t^{2} s_{2} / s u-5 t s_{2} / s\right] / 2 ; \quad \Delta \tilde{e}_{7}=-2 m^{2} \\
\Delta e_{8} & =4 m^{4}(s / t u+2 / u+1 / s)-2 m^{2}\left(s^{2} / t u+2 s_{2} / u-1+t / s\right)-\left(s^{2} / t+s+3 s_{2}\right. \\
& \left.+t^{2} / s+3 t u / s\right) s / u ; \quad \Delta e_{9}=8\left(1 / u-1 / s_{2}\right) / t \\
\Delta e_{10} & =4\left[2\left(1 / u+2 s / s_{2} t-2 / s_{2}\right) m^{2} / u+s_{2} / t u-3 / s_{2}-u / t s_{2}\right] \\
\Delta \tilde{e}_{11} & =8 m^{4}\left(s / t+t / u+s_{2} / s\right)-2 m^{2}\left[2 s^{2} / u+2 s^{2} / t+s+s_{2}(2 t / u+2+4 u / t+t / s+u / s)\right] \\
& -\left(s^{2}+t^{2}\right)(t+u) / u ; \quad \Delta \tilde{e}_{12}=m^{2} s ; \quad \Delta e_{13}=-m^{2}\left(4 m^{2} s / t+2 s+u\right) \\
\Delta \tilde{e}_{14} & =-m^{4} s ; \quad \Delta e_{15}=4\left[2 m^{2} / u-2 m^{2} / t-t / u+u / t\right] / s^{2} \\
\Delta e_{16} & =a_{1}+a_{1}(t \leftrightarrow u)-8\left(m^{2}+m^{2} t / s-t\right) / s u+2\left[t^{2}(t / u+2)-u^{2}(u / t+2)\right] / s^{2} s_{2}
\end{aligned}
$$

where

$$
a_{1}=t\left[4 m^{2}(7 s / t+1+3 t / s+9 u / s)-2 s+6 s u / t+4 t+u\right] / s_{2} u s
$$

For $|M|_{2 \rightarrow 3}^{2}$ given in Eq. 24 and $d \sigma_{\mathrm{Br}} / d v d w$ given in Eq. 30, the coefficients $e_{i}$ are

$$
\begin{aligned}
\tilde{e}_{1} & =2\left[16 m^{6}(t+u) / t u+16 m^{4}\left(s_{2} / u+s / t+3\right)+2 m^{2}\left(25 s_{2}-2 t+2 s_{2}^{2} / u\right)\right. \\
& \left.+s_{2}(2 s+5 t+10 u)\right] / u ; \quad e_{2}=-8\left[2 m^{2}(t+u) / s_{2} t+3\right] / t u \\
e_{3} & =-2\left[16 m^{6}(t+u) / s_{2} t u+8\left(4+u / t-2 s / s_{2}\right) m^{4} / u+2 m^{2}\left(6 t / u+6-u / t-s / t+s / s_{2}\right)\right. \\
& \left.-2 s^{2} / u-3 s t / u-2 s-s_{2}+3 s t / s_{2}-2 t^{3} / s_{2} u\right] / t \\
e_{4} & =2 m^{2}\left(2 m^{2} / t+1\right)^{2} ; \quad e_{5}=-4 m^{2} / u \\
\tilde{e}_{6} & =-16 m^{4} / u+2 m^{2}(5 s / u+7 t / u+11)+t(4 s / t-s / u+t / u+17) ; \quad \tilde{e}_{7}=2 m^{2} \\
e_{8} & =8 m^{6}(s / t+2+u / s) / s u+4\left(3 s / t+4+2 s_{2} / s\right) m^{4} / u+2 m^{2}\left(s_{2} / u-2-t / s\right) \\
& -\left(s^{2} / t+s+3 s_{2}+t^{2} / s+3 t u / s\right) s / u ; \quad e_{9}=8\left(s_{2}+u\right) / t s_{2} u \\
e_{10} & =-4\left[8 m^{4} / u+2 m^{2}(s / u+2)-s_{2}^{2} / u+4 s+t+3 u\right] / t s_{2} \\
\tilde{e}_{11} & =16 m^{6}\left[(t+u)^{2} / t u+s_{2} / s\right] / s+8 m^{4}\left[(s-t) / u+t^{2} / s u+3(t+u) / s+u^{2} / s t\right] \\
& +2 m^{2}\left[u(s+u) / t+t(t+u) / s-t s_{2} / u-7 s_{2}-2 t s_{2} / s-u s_{2} / s\right]+\left(s^{2}+t^{2}\right)(t+u) / u \\
\tilde{e}_{12} & =m^{2}\left(8 m^{4} / s-4 m^{2}-s\right) ; \quad e_{13}=m^{2}\left(8 m^{4} / t+4 m^{2}-t\right) ; \quad \tilde{e}_{14}=2 m^{6} ; \quad e_{15}=0 \\
e_{16} & =-2\left[22 m^{2}(t+u) / t u+3 t / u+14+u / t\right] / s_{2}
\end{aligned}
$$




\section{APPENDIX C}

We give here the bremsstrahlung integrals, $I_{i}$, appearing in Eq. (30). They are defined as

$$
I_{i}=\frac{1}{2 \pi} \int d \Omega f_{i} ; \quad(2 m)^{2}[\Delta]|M|_{2 \rightarrow 3}^{2} \equiv C \sum_{i}[\Delta] e_{i} f_{i} / s_{2}^{n_{i}}
$$

(see (24)). The $f_{i}$ may be explicitly expressed as functions of $\theta_{1}$ and $\theta_{2}$ using the expressions in Appendix A. All the integrals here are 4-dimensional (i.e. $\varepsilon=0$ in (27)) and are determined using the general forms given in Ref. 2 .

First we list the four basic integrals,

$$
\begin{aligned}
I_{6} & =\frac{2 S_{2}}{s_{2} \bar{y}} \ln \frac{T+U-\bar{y}}{T+U+\bar{y}}, \quad I_{6}^{\prime} \equiv \frac{2 S_{2}}{s_{2}(s+t)} \ln \frac{S_{2}}{m^{2}} \\
I_{8} & =\frac{4 S_{2}}{s_{2} \sqrt{s}} \frac{1}{\sqrt{x_{8}}} \ln \frac{x_{8}+s_{2}^{2} s+2 s_{2} \sqrt{s x_{8}}}{x_{8}+s_{2}^{2} s-2 s_{2} \sqrt{s x_{8}}}, \quad x_{8} \equiv 4 m^{2}\left(s_{2} s+t u\right)+s_{2}^{2} s \\
I_{11} & =\frac{4 S_{2}}{s_{2} \sqrt{s t x_{11}}} \ln \frac{x_{11}+s t-2 \sqrt{s t x_{11}}}{x_{11}+s t+2 \sqrt{s t x_{11}}}, \quad x_{11} \equiv 4 m^{2}\left(s_{2}-t\right)+s t
\end{aligned}
$$

Define,

$z_{1} \equiv 2 m^{2} s+s_{2} s-t u, \quad z_{2} \equiv s_{2} u-2 m^{2} s-s t, \quad z_{3} \equiv m^{2} s-t u, \quad z_{4} \equiv 2 m^{2} s-t u, \quad z_{5} \equiv 2 m^{2}+t$

We may now express the remaining integrals in terms of those listed above:

$$
\begin{aligned}
I_{5} & =-\frac{2 S_{2} z_{4}}{m^{2}(s+t)^{3}}+\frac{I_{6}^{\prime} z_{1}}{(s+t)^{2}} \\
I_{9} & =\frac{1}{4 S_{2}(s+t)^{3}}\left\{2 z_{1}\left(s_{2}-t\right)(s+t) s_{2}-\left(z_{1}^{2}+2 z_{3} S_{2} s\right)\left(2 m^{2}+s_{2}\right)\right\}+\frac{I_{6}^{\prime}}{4(s+t)^{2}}\left(z_{4}^{2}+2 m^{2} s z_{3}\right) \\
I_{10} & =\frac{z_{1}}{(s+t)^{2}}-\frac{I_{6}^{\prime} z_{4}}{2(s+t)} ; \quad I_{12}=\frac{8 S_{2}}{m^{2} s_{2} t}\left(\frac{z_{5}}{x_{11}}-\frac{1}{s_{2}}\right)-2 I_{11} \frac{z_{5}\left(s_{2}-t\right)}{x_{11} t} \\
I_{13} & =-\frac{8 S_{2} z_{5}}{x_{11}\left(s_{2}-t\right) m^{2} t}-2 \frac{I_{11}}{t}\left(1-\frac{s_{2} z_{5}}{x_{11}}\right) \\
I_{14} & =\frac{16 S_{2}}{s t x_{11}}\left(\frac{12 z_{3}}{x_{11} t}+\frac{\bar{y}^{2}+s_{2}^{2}}{s_{2}^{2} m^{2}}\right)-\frac{4 I_{11}}{x_{11} s t}\left\{s_{2} u-s z_{5}-3 z_{5}\left(s_{2}-t\right) \frac{s}{t}\left(1-\frac{s_{2} z_{5}}{x_{11}}\right)\right\} \\
I_{15} & =\frac{s_{2}}{4 S_{2} \bar{y}^{4}}\left\{\left(z_{2}^{2}+2 S_{2} s z_{3}\right)(U+T)+2 \bar{y}^{2} z_{2}\left(s_{2}-t\right)\right\}+\frac{I_{6} s_{2}^{2}}{4 \bar{y}^{4}}\left\{\left(z_{4}-u^{2}\right)^{2}+2 m^{2} s z_{3}\right\} \\
I_{16} & =\frac{z_{2}}{\bar{y}^{2}}-\frac{I_{6} s_{2}\left(z_{4}-u^{2}\right)}{2 \bar{y}^{2}}
\end{aligned}
$$

The integrals were put into the above form using REDUCE. The integrals not listed here (including the $n$-dimensional ones not given in Ref. 2) are straightforward and have been 
substituted directly in (30). As an aside, we point out that $x_{11}(t \leftrightarrow u)$ vanishes for $v=1 / 2$, $w=w_{1}$. Hence one must avoid reaching exactly the lower bound (as for the upper) of the $w$ integral, in numerical calculations.

\section{REFERENCES}

* On leave from High Energy Physics Institute, Tbilisi State University, Tbilisi, Republic of Georgia.

1. P. Nason, S. Dawson, and R.K. Ellis, Nucl. Phys. B303, 607 (1988); B327, 49 (1989) and erratum B335, 260 (1990).

2. W. Beenakker, H. Kuijf, W.L. van Neerven, and J. Smith, Phys. Rev. D40, 54 (1989).

3. J. Smith and W.L. van Neerven, Nucl. Phys. B374, 36 (1992); J.H. Kühn, E. Mirkes, and J. Steegborn, Z. Phys. C57, 615 (1993); M. Drees, M. Krämer, J. Zunft, and P.M. Zerwas, Phys. Lett. 306B, 371 (1993).

4. J.F. Gunion and H.E. Haber, Phys. Rev. D48, 5109 (1993).

5. D.L. Borden, V.A. Khoze, J. Ohnemus, and W.J. Stirling, Phys. Rev. D50, 4499 (1994).

6. W. Siegel, Phys. Lett. 84B, 193 (1979).

7. J.C. Ward, Phys. Rev. 78, 182 (1950).

8. A.P. Contogouris, B. Kamal, and Z. Merebashvili, Phys. Lett. 337B, 169 (1994).

9. A.P. Contogouris, O. Korakianitis, F. Lebessis, and Z. Merebashvili, Phys. Lett. B344, 370 (1995).

10. G.A. Schuler, S. Sakakibara, and J.G. Körner, Phys. Lett. 194B, 125 (1987); J.G. Körner and M.M. Tung, Z. Phys. C64, 255 (1994).

11. I. Jack, D.R.T. Jones and K.L. Roberts, Z. Phys. C62, 161 (1994); C63, 151 (1994); M. Ciuchini, E. Franco, L. Reina, and L. Silvestrini, Nucl. Phys. B421, 41 (1994).

12. G. Passarino and M. Veltman, Nucl. Phys. B160, 151 (1979).

13. J. Vermaseren, FORM User's Manual (CAN, Amsterdam, 1991).

14. A.C. Hearn, REDUCE User's Manual Version 3.3 (Rand Corporation, Santa Monica, CA, 1987).

15. R.K. Ellis, M.A. Furman, H.E. Haber, and I. Hinchliffe, Nucl. Phys. B173, 397 (1980).

16. CDF Collaboration, F. Abe et al., Phys. Rev. Lett. 73, 225 (1994); Phys. Rev. D50, 2966 (1994). 


\title{
Erratum: Heavy-quark production by polarized and unpolarized photons in next-to-leading order [Phys. Rev. D 51, 4808 (1995)]
}

\author{
B. Kamal, Z. Merebashvili and A.P. Contogouris
}

PACS number(s): 13.65.+i, 13.88.+e, 14.65.-q, 14.70.Bh

Due to a trivial algebraic error, Eq. (30) is incorrect. On the fourth line one should make the replacement $-1 \rightarrow 2 \ln x$. This gives for Eq. (30)

$$
\begin{aligned}
\frac{d \sigma_{\mathrm{Br}}}{d v d w} & =\cdots+8 \pi \alpha_{s} C_{F} C_{\varepsilon} \frac{d \sigma_{\mathrm{LO}}}{d v d w} \frac{1}{s \beta}\left(( 2 m ^ { 2 } - s ) \left\{2 \ln x\left(2 \ln \frac{s v}{m^{2}}-\frac{1}{\varepsilon}+1\right)\right.\right. \\
& \left.\left.-2\left[\operatorname{Li}_{2}\left(\frac{-4 \beta}{(1-\beta)^{2}}\right)+\ln ^{2} x\right]\right\}+2 s \beta\left[1-2 \ln \frac{s v}{m^{2}}+\frac{1}{\varepsilon}\right]\right) .
\end{aligned}
$$

The essential conclusions remain unchanged as the features of the figures are the same. Various checks were done to ensure that the analytical results (not completely presented in any other works) are now correct. We now agree exactly with Table 1 of Kühn et. al. [3] and the corresponding polarized results in Table 1 of [G. Jikia and A. Tkabladze, Phys. Rev. D 54, 2030 (1996)]. We also agree exactly with the unpolarized squared amplitude of [J.F. Gunion and Z. Kunszt, Phys. Lett. B 178, 296 (1986)] (although our 3-jet cross sections had a minor numerical error which made the ones presented in Figures 4 and 5 a bit too large on average) and all the bremsstrahlung integrals, including the $n$-dimensional ones, have been checked numerically. Finally, we agree exactly with the unpolarized virtual+soft cross section of [2] and consequently obtain the correct threshold behavior. The soft part of [2] may be obtained by making the substitution $\ln \left(s v / m^{2}\right) \rightarrow \ln \delta$ in the above expression, as may be easily derived. 